\title{
Increasing efficiency through optimal RK time integration of diffusion equations
}

\author{
Fausto Cavalli ${ }^{1}$, Giovanni Naldi ${ }^{1}$, Gabriella Puppo $^{2}$, and Matteo Semplice ${ }^{1}$ \\ 1 Dipartimento di Matematica, Università di Milano, via Saldini 50, 20133 Milano, \\ ITALY. \{cavalli, naldi, semplice\}@mat.unimi.it \\ 2 Dipartimento di Matematica, corso Duca degli Abruzzi, 24, 10129 Torino, Italy \\ gabriella.puppo@polito.it
}

Summary. The application of Runge-Kutta schemes designed to enjoy a large region of absolute stability can significantly increase the efficiency of numerical methods for PDEs based on a method of lines approach. In this work we investigate the improvement in the efficiency of the time integration of relaxation schemes for degenerate diffusion problems, using SSP Runge-Kutta schemes and computing the maximal CFL coefficients. This technique can be extended to other PDEs, linear and nonlinear, provided the space operator has eigenvalues with a non-zero real part.

\section{Introduction}

The integration of evolution PDE's through a method of lines approach leads to the solution of large systems of ODE's. Often such ODE's are stiff or moderately stiff; therefore the possibility of increasing the stability region of the time integrator can lead to a significant increase in efficiency for explicit or semi-implicit time-integration.

Specifically, we consider the system of PDE's

$$
u_{t}+f_{x}(u)=D p_{x x}(u)
$$

where $f(u)$ is hyperbolic, i.e. the Jacobian of $f$ is provided with real eigenvalues and a basis of real eigenvectors for each $u$, while $p(u)$ is a non decreasing Lipshitz continuous function, with Lipshitz constant $\mu$. We assume the system has been fully discretized in space on a grid of $N$ points $x_{j}, j=1, \ldots N$, and we denote with $U(t)=\left[U_{1}(t), \ldots, U_{N}(t)\right]^{T}$ the vector of the grid values of the numerical solution at time $t$. The space discretized system can be written in the form:

$$
\frac{d U}{d t}=L(U(t))
$$

leading to an autonomous system of $N$ non linear first order ODE's. If we consider for instance a system of conservation laws, $D=0$, the operator $L$ obtained from a conservative space discretization, will be written as: 


$$
L(U)=-\frac{1}{h}\left(F_{j+1 / 2}-F_{j-1 / 2}\right),
$$

where $F_{j+1 / 2}$ is the numerical flux consistent with the physical flux $f(u)$ in the usual sense of the Lax-Wendroff theorem and $h$ is the grid spacing.

In recent years, much research has focused on the efficient integration of the semidiscrete system (2). In particular, in this work we will concentrate on the performance of optimal Runge-Kutta schemes, characterized by a large stability region, introduced in [SR02].

Optimal Runge-Kutta schemes are built choosing an accuracy order $p$ and a number of stages $s$, with $s \geq p$. In principle, once $s$ and $p$ are fixed, the coefficients of the Butcher tableaux defining the Runge-Kutta schemes are computed maximizing in some sense the stability region and keeping as constraints the fulfilment of the accuracy requirements. To compute their optimal schemes, Spiteri and Ruuth in [SR02] start from a strong stability condition which requires that the operator $L$ be non linearly stable with respect to a suitable norm for a certain CFL with Forward Euler integration, namely:

$$
\left\|U^{n}+\Delta t L\left(U^{n}\right)\right\| \leq\left\|U^{n}\right\|, \quad \forall \Delta t \leq \Delta t_{F E} .
$$

Once this assumption is satisfied, the optimal schemes proposed in [SR02] do yield considerable savings in CPU time for a given accuracy.

The idea is that an $s$ stages Runge-Kutta scheme applied to (2) can be written as a convex combination of $s$ Forward Euler steps as (see also [GST01]):

$$
\begin{aligned}
U^{(1)} & =U^{n} \\
U^{(i)} & =\sum_{k=1}^{i-1} \alpha_{i k}\left[U^{(k)}+\Delta t \frac{\beta_{i k}}{\alpha_{i k}} L\left(U^{(k)}\right)\right] \\
U^{(n+1)} & =U^{(s)} .
\end{aligned}
$$

Thus if the space discretized operator $L$ is strongly stable for $\Delta t \leq \Delta t_{F E}$ with Forward Euler time integration, than the scheme in (5) will be strongly stable for:

$$
\Delta t \leq \lambda \Delta t_{F E}, \quad \lambda=\min \frac{\alpha_{i k}}{\beta_{i k}}
$$

Note that (6) is only a sufficient condition for stability: clearly it may be possible to violate (6) and still find a stable scheme.

The problem is that several high order space discretization operators $L$ are not stable under the Forward Euler scheme, and therefore it is not possible to use the estimate (6) to guarantee that optimal SSP schemes will improve the efficiency of the time integration of (2).

In the case of pure convection, that is for $D=0$ in (1), high order space discretizations of $f_{x}(u)$ have purely imaginary eigenvalues $\nu$ up to high order powers of the mesh width $h$, that is $\Re(\nu)=O(h)^{p}$ and therefore are not 
stable under Forward Euler time integration. This is the case for instance of the fifth order WENO space discretization, see also [Spi], but we conjecture that the same holds for other widely used high order space discretizations for convective operators, such as ENO.

On the other hand, when $D \neq 0$, the eigenvalues of the semidiscrete operator $L$ do have a negative real part of order 1 , and therefore can be made stable under Forward Euler for a non zero $\Delta t_{F E}$. In this work we study the stability of a family of high order numerical fluxes coupled with optimal RK-SSP schemes for (1), in the case of pure diffusion, i.e. $f \equiv 0$. In this case we find that several widespread space discretization schemes are stable with Forward Euler time integration, and therefore optimal RK-SSP schemes do yield a significant increase in the allowable CFL. Since in this case the eigenvalues of the exact operator are real, we even find that the stability estimate in (6) may be quite pessimistic, because it underestimates the stability region of some SSP schemes. In these cases, it is easy to compute numerically the maximal CFL, obtaining a further improvement in the efficiency of the scheme.

\section{Diffusive relaxation}

We consider high order approximations of the degenerate parabolic equation

$$
u_{t}=D p_{x x}(u),
$$

using relaxation schemes, a technique initially proposed in [JX95]. Following [NP00] and [CNPS06], we rewrite the system as a hyperbolic system with a stiff source term depending on a parameter $\varepsilon$, which formally relaxes on the original parabolic equation as $\varepsilon \rightarrow 0$, namely:

$$
\left\{\begin{array}{l}
\frac{\partial u}{\partial t}+\frac{\partial v}{\partial x}=0 \\
\frac{\partial v}{\partial t}+\Phi^{2} \frac{\partial w}{\partial x}=-\frac{1}{\varepsilon} v+\left(\Phi^{2}-\frac{D}{\varepsilon}\right) \frac{\partial w}{\partial x} \\
\frac{\partial w}{\partial t}+\frac{\partial v}{\partial x}=-\frac{1}{\varepsilon}(w-p(u))
\end{array}\right.
$$

where $\Phi^{2}$ is a suitable positive parameter. Formally, as $\varepsilon \rightarrow 0, w \rightarrow p(u)$, and $v \rightarrow-\partial_{x} p(u)$ and the original equation (7) is recovered. We integrate the system above with an IMEX Runge-Kutta scheme [PR05]. In this fashion, the stiff source term is implicit and does not require restrictive stability conditions, while the linear convective term is explicit.

In this work we consider only the relaxed scheme, which is obtained setting $\varepsilon=0$ in the discretized equations. Let $\tilde{a}_{i, k}$ and $\tilde{b}_{i}$ be the coefficients forming the Butcher tableaux of the explicit Runge-Kutta scheme in the IMEX pair. 
The computation of the first stage value of the Runge Kutta scheme reduces to:

$$
\begin{aligned}
& u^{(1)}=u^{n} \\
& w^{(1)}=p\left(u^{n}\right) \\
& v^{(1)}=-D \partial_{x} w^{(1)}
\end{aligned} .
$$

For the following stages the first equation is

$$
u^{(i)}=u^{n}-\Delta t \sum_{k=1}^{i-1} \tilde{a}_{i, k} \partial_{x} v^{(k)} .
$$

In the other equations the convective terms are dominated by the source terms and thus $v^{(i)}$ and $w^{(i)}$ are given by

$$
w^{(i)}=p\left(u^{(i)}\right), \quad v^{(i)}=-D \partial_{x} w^{(i)} .
$$

In this fashion, due to the particular structure of the relaxation scheme we are considering, only the explicit tableaux of the Implicit-Explicit Runge-Kutta scheme enters the actual computation, while the coefficients of the implicit scheme drop out as the relaxation step is computed. For this reason we can apply any explicit Runge-Kutta scheme to advance in time the solution $u$. Finally the updated solution is given by:

$$
u^{n+1}=u^{n}-\Delta t \sum_{i=1}^{\nu} \tilde{b}_{i} \partial_{x} v^{(i)}
$$

The overall accuracy of the scheme depends on the accuracy of the numerical flux used to approximate $\partial_{x} v^{(i)}$ and on the accuracy of the Runge-Kutta explicit scheme.

A non linear stability analysis for the first order version of this scheme (upwind numerical flux on a piecewise constant space reconstruction to evaluate $\partial_{x} v$ and Forward Euler in time) yields a parabolic stability restriction of the form:

$$
\Delta t \leq \frac{2 h^{2}}{\mu} \frac{1}{1+2 h \phi}
$$

where $\mu$ is the Lipshitz continuity constant of $p(u)$, see [CNPS06]. The parabolic CFL implies that to obtain a scheme of order $p$ one should use a $p$ th order accurate numerical flux for $\partial_{x} v$ and a $p / 2$ order accurate Runge-Kutta method in time.

A linear stability analysis of several higher order numerical fluxes yields again a parabolic CFL, of the form $\Delta t \leq C_{1} h^{2}\left(1-C_{2} h \Phi\right) / \mu$, with constant $C_{1}$ given by Table 1 . Note that the scheme is unstable for $\Delta t=C_{1} h^{2} / \mu$, but for a sufficiently small $h$ it is enough to pick $\Delta t=\left(C_{1}-\delta\right) h^{2} / \mu$ for a suitable positive $\delta$, i.e. the scheme is stable provided $C_{1}$ is slightly decreased, see Table 3 . Note that the stability requirement becomes more strict as space accuracy increases, while it loosens as time accuracy increases. 


\begin{tabular}{|c|c|c|c|}
\hline & RK1 & RK2 & RK3 \\
\hline P-wise constant & 2 & 2 & 2.51 \\
\hline P-wise linear & 0.94 & 0.94 & 1.18 \\
\hline WENO5 & 0.79 & 0.79 & 1 \\
\hline
\end{tabular}

Table 1. CFL constant $C_{1}$ for a few space reconstruction algorithms (from linear analysis) for standard first, second and third order RK schemes

\section{Diffusive relaxation and SSP schemes}

We start fixing a standard notation for $s$ stages Runge-Kutta schemes. As in [SR02] we denote SSP(s,p) the optimal strongly stable Runge-Kutta scheme of order $p$ with $s$ stages. We point out that $\operatorname{SSP}(1,1)$ is the Forward Euler scheme, $\operatorname{SSP}(2,2)$ the Heun scheme and $\operatorname{SSP}(3,3)$ the TVD third order Runge-Kutta method of [SO88], which is probably the high order Runge-Kutta scheme most frequently used in conjunction with high order space discretizations for (1).

\begin{tabular}{|c|c|c|c|c|c|}
\hline & $\mathrm{s}=1$ & $\mathrm{~s}=2$ & $\mathrm{~s}=3$ & $\mathrm{~s}=4$ & $\mathrm{~s}=5$ \\
\hline $\mathrm{p}=1$ & 1 & 2 & 3 & 4 & 5 \\
\hline $\mathrm{p}=2$ & & 1 & 2 & 3 & 4 \\
\hline $\mathrm{p}=3$ & & & 1 & 2 & 2.65 \\
\hline
\end{tabular}

Table 2. Improved stability coefficients for several $s$ stages order $p$ Runge-Kutta schemes (from [SR02])

From the first column of Table 1 we note that all the numerical fluxes considered are at least linearly stable with the Forward Euler scheme. Thus the theory of strongly stable Runge-Kutta schemes can be applied in this case. In particular we consider the schemes introduced in [SR02], for which the improved stability coefficients $\lambda$ of (6) can be found in Table 2. To indicate the fact that these coefficients are found through the theory of strongly stable Runge Kutta schemes and to identify to which scheme they apply, we will label these coefficients as $\lambda_{\mathrm{SSP}}(s, p)$.

The coefficients $\lambda_{\mathrm{SSP}}(s, p)$ are not optimal in the case of pure diffusion. They can be further improved considering the actual stability region of SSP schemes and recalling that for the purely diffusive operator we are considering, only the intersection of the stability region with the real line is relevant.

In Figure 1 we find the regions of absolute stability for some SSP schemes given in [SR02]. On the top left of the figure we find the stability regions of schemes of order 1 with 1,2 and 3 stages. We point out that here the improvement in the CFL constant is exactly balanced by the increased computational effort due to the higher number of stages: there is no gain in efficiency with respect to the standard Forward Euler scheme. In the top right of Figure 1 

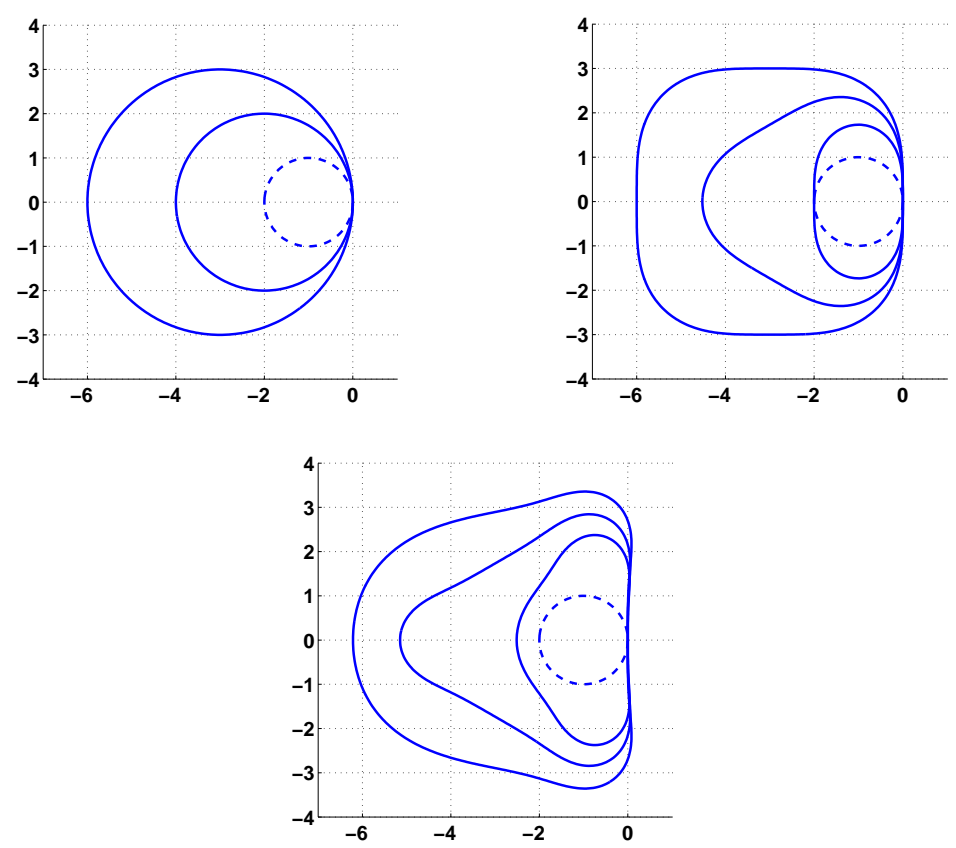

Fig. 1. Regions of absolute stability for optimal SSP schemes. On the top left: order 1 , stages $1,2,3$. On the top right: order 2 , stages $2,3,4$. On the bottom: order 3 , stages $3,4,5$. The dashed line is the Forward Euler scheme.

there are the stability regions of schemes of order 2 with 2, 3 and 4 stages and, for comparison, of the Forward Euler scheme (dashed line). The SSP theory underestimates the effective CFL and direct inspection of the stability plot suggests that the stability coefficient $\lambda$ appearing in Table 3 can be increased finding the intersection of the stability curve with the real axis. Finally, on the bottom of Figure 1 we show the stability regions of schemes of order 3 with 3,4 and 5 stages. Again the CFL gain of Table 2 can be improved by direct inspection of the graph.

Let $\eta_{s, p}$ be the abscissa of the intersection of the stability curve with the negative real axis. Then the maximal gain in CFL with respect to Forward Euler, for a problem with real eigenvalues, is given by

$$
\lambda_{\mathrm{OPT}}(s, p)=\frac{\left|\eta_{s, p}\right|}{\left|\eta_{1,1}\right|}=\frac{\left|\eta_{s, p}\right|}{2}
$$

For several schemes in the figure it is easy to see that $\lambda_{\mathrm{OPT}}(s, p)>\lambda_{\mathrm{SSP}}(s, p)$.

The optimal CFL number for a given scheme can be found multiplying the coefficient $C_{1}-\delta$ determined by the space discretization by the proper stability coefficient $\lambda$, i.e. $\Delta t \leq\left(C_{1}-\delta\right) \lambda_{\mathrm{OPT}}(s, p) h^{2} / \mu$. 


$\operatorname{SSP}(2, \mathrm{~s})+$ WENO 5
\begin{tabular}{|c|c|c|c|}
\hline stages & CFL & order & $N_{f}$ \\
\hline 2 & 0.78 & 4 & 810 \\
\hline 3 & $2 \times 0.78$ & 4 & 606 \\
\hline 4 & $3 \times 0.78$ & 4 & 540 \\
\hline
\end{tabular}
\begin{tabular}{|c|c|c|c|}
\hline SSP $(3, \mathrm{~s})+$ WENOS & CFL & order & $N_{f}$ \\
\hline 3 & 0.78 & 4.5 & 1230 \\
\hline 4 & $2 \times 0.78$ & 5.2 & 820 \\
\hline 5 & $2.65 \times 0.78$ & 5.2 & 770 \\
\hline
\end{tabular}

\begin{tabular}{|c|c|c|c|}
\hline \multicolumn{4}{|c|}{$\operatorname{SSP}(2, \mathrm{~s})+\mathrm{WENO} 5$} \\
\hline stages & \begin{tabular}{|c|}
$\mathrm{CFL}$ \\
\end{tabular} & order & $\overline{N_{f}}$ \\
\hline 2 & $1 \times 0.78$ & 4 & 810 \\
\hline 3 & $2.259 \times 0.78$ & 4 & $\overline{537}$ \\
\hline 4 & $3 \times 0.78$ & 4 & 540 \\
\hline \multicolumn{4}{|c|}{$\overline{\mathrm{SSP}(3, \mathrm{~s})+\mathrm{WENO} 5}$} \\
\hline stages & CFL & order & $\overline{N_{f}}$ \\
\hline 3 & $\mathbf{1 . 2 5 6} \times 0.78$ & 4.8 & 978 \\
\hline 4 & $\mathbf{2 . 5 7 4 \times 0 . 7 8}$ & $\overline{5.6}$ & 636 \\
\hline 5 & $\mathbf{3 . 1 0 6} \times 0.78$ & 5.4 & 660 \\
\hline
\end{tabular}

Table 3. Order of convergence and number of numerical flux function evaluations $N_{f}$ (with 80 grid points) for the SSP-CFL $\lambda_{\mathrm{SSP}}(s, p)$ (left) and the maximal CFL $\lambda_{\mathrm{OPT}}(s, p)$ (right) for several RK schemes.

To measure the computational complexity of a scheme we compute the number $N_{f}$ of numerical flux evaluations needed to reach a fixed integration time. Thus, for a given numerical flux, the most efficient scheme has the lowest value of $N_{f}$. Table 3 shows the different values of $N_{f}$ obtained with CFL chosen according to the values of $\lambda_{\mathrm{SSP}}(s, p)$ on the left and $\lambda_{\mathrm{OPT}}(s, p)$ on the right. The grid spacing $h$ is the same for all values of $N_{f}$, namely, $h=1 / 80$. The table contains also data on the accuracy of the space-time scheme. The accuracy was evaluated using four different grids, and modifying the time step according to $h$ and the chosen value of $\lambda$. We don't show data for first order schemes since $\lambda_{\mathrm{SSP}}(s, 1)=\lambda_{\mathrm{OPT}}(s, 1)$. The table shows the increased efficiency of the $\operatorname{SSP}(\mathrm{s}, \mathrm{p})$ schemes with $s>p$ (left column) and the gain obtained by a better estimate of $\lambda$ (right column). In the table, the optimal values of $\lambda$ are indicated in bold face, when they are sensitively larger than the corresponding $\lambda_{\mathrm{SSP}}(s, p)$. In particular, the improvement between the standard $\operatorname{SSP}(3,3)$ and the five stages $\operatorname{SSP}(5,3)$ with the optimal CFL is quite striking.

The data in Table 3 refer to a linear diffusion problem, although we find analogous results for a nonlinear degenerate diffusion equation. We performed tests on the self-similar Barenblatt solution of the porous media equation. In this case the order of accuracy is limited by the non regularity of the solution, but we find that the errors with respect to the exact solution slightly decrease using the $\operatorname{SSP}(\mathrm{s}, \mathrm{p})$ with $s>p$ and the optimal $\lambda$.

\section{Final remarks}

We have shown that the theory of [SR02] can be applied to diffusion equations in the relaxation framework to improve the efficiency of the time integration. Moreover the fact that the eigenvalues of the semidiscrete operator are real numbers permits to further improve the efficiency of the schemes. We expect that analogous results can be obtained for convection-diffusion operators, 
thanks to the nonzero real part of the eigenvalues of the discrete operators. This allows to achieve stability under Forward Euler integration.

We also note that the same framework can be applied to other space discretizations besides the numerical fluxes obtained via relaxation schemes: the key factor is the localization of the eigenvalues of the differential operator, which must have a non zero real part. On the other hand, the plots of absolute stability regions show that Runge-Kutta schemes with number of stages $s>p$ can be built with improved stability regions, notwithstanding stability under Forward Euler. These schemes can be applied to semidiscrete operators even in the convective regime, but their stability conditions cannot be derived from the behaviour of the operator under the Forward Euler scheme.

\section{References}

[CNPS06] F. Cavalli, G. Naldi, G. Puppo, and M. Semplice. High order relaxation schemes for non linear diffusion problems. 2006. http://arxiv.org/abs/math?papernum=0604572. Submitted to SINUM.

[GST01] S. Gottlieb, C. Shu, and E. Tadmor. Strong stability-preserving high-order time discretization methods. SIAM Rev., 43(1):89-112, 2001.

[JX95] S. Jin and Z. Xin. The relaxation schemes for systems of conservation laws in arbitrary space dimension. Comm. Pure and Appl. Math., 48:235-276, 1995.

[NP00] G. Naldi and L. Pareschi. Numerical schemes for hyperbolic systems of conservation laws with stiff diffusive relaxation. SIAM J. Numer. Anal., 37:1246-1270, 2000.

[PR05] L. Pareschi and G. Russo. Implicit-explicit Runge-Kutta schemes and applications to hyperbolic systems with relaxation. J. Sci. Comp., 25:129$155,2005$.

[SO88] Chi-Wang Shu and Stanley Osher. Efficient implementation of essentially nonoscillatory shock-capturing schemes. J. Comput. Phys., 77(2):439-471, 1988.

[Spi] Raymond J. Spiteri. Private communication.

[SR02] Raymond J. Spiteri and Steven J. Ruuth. A new class of optimal highorder strong-stability-preserving time discretization methods. SIAM J. Numer. Anal., 40(2):469-491 (electronic), 2002. 